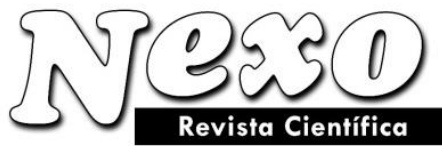

ISSN-E 1995-9516

Universidad Nacional de Ingeniería COPYRIGHT () (UNI). TODOS LOS DERECHOS RESERVADOS http://revistas.uni.edu.ni/index.php/Nexo https://doi.org/10.5377/nexo.v34i05.13109

\title{
Efecto del agregado grueso triturado en las propiedades del concreto
}

\section{Effect of crushed coarse aggregate on properties of concrete}

\author{
Eddisson Francisco Hernández \\ Universidad Nacional de Ingeniería. Facultad de Arquitectura/Facultad de Tecnología de la Construcción. \\ Managua, Nicaragua. \\ *eddison.hernandez@uni.edu.ni
}

(recibido/received: 03-octubre-2021; aceptado/accepted: 23-noviembre-2021)

\section{RESUMEN}

Este trabajo presenta el efecto del uso de agregado grueso triturado de diferentes tamaños y calidades, sobre las propiedades mecánicas del concreto. Se elaboraron mezclas de concreto con relaciones agua/cemento de 0.50 y 0.45 , con dos tipos de cementos y agregado grueso de 1/2" y 3/4", calidad A y B. Se elaboraron especímenes cilíndricos $(100 \mathrm{~mm}$ x $200 \mathrm{~mm})$ y curaron en húmedo por 7 días. Se determinó la resistencia a la compresión y velocidad de pulso ultrasónico a diferentes edades. Los resultados muestran que el tamaño y la calidad del agregado afectaron la resistencia mecánica de las mezclas de concreto.

Palabras claves: Cemento; agregado grueso; resistencia a la compresión; velocidad de pulso ultrasónico.

\begin{abstract}
This paper presents the effect of the use of crushed coarse aggregate of different size and qualities, on the mechanical properties of concrete. Concrete mixtures with water/cement ratios of 0.50 and 0.45 , with two types of cement, and coarse aggregates of $1 / 2$ " and $3 / 4$ ", quality A and B, were prepared. Cylindrical specimens $(100 \mathrm{~mm} \times 200 \mathrm{~mm})$ were cast and moist-cured for 7 days. The compressive strength and the ultrasonic pulse velocity at different ages were determinate. Results indicate that the size and quality of aggregate affected the mechanical resistance of concrete mixtures.
\end{abstract}

Keywords: Cement; coarse aggregate; compressive strength; ultrasonic pulse velocity.

\section{INTRODUCCIÓN}

El concreto es un material compuesto que consiste esencialmente de agregados, agua y cemento, los cuales, en estado endurecido forman una masa similar a una roca (Kosmatka et al., 2004; Mehta y Monteiro, 2006). Los agregados utilizados en la elaboración de concreto son arena (natural o artificial) y grava (natural o piedra triturada) con una resistencia mecánica adecuada, que constituyen aproximadamente del $60 \%$ al $75 \%$ del volumen total de la mezcla (Kosmatka et al., 2004). Las propiedades de los agregados tales como la porosidad, granulometría, absorción, peso, tamaño, forma y textura, densidad, propiedades térmicas, así como la zona de transición interfacial (contacto entre la pasta de cemento y el agregado), juegan un papel importante en la resistencia y durabilidad del concreto (Mehta y Monteiro, 2006). 
En mezclas de concreto, la densidad de los agregados determinan la densidad del material, así como la porosidad produce concretos de baja resistencia mecánica (Muhit et al., 2013). La redondez y angularidad de los agregados son características importantes que afectan las propiedades del concreto. Partículas redondeadas y bien graduadas, presentan un menor contenido de vacíos en comparación con agregados alargados y angulares. Para el caso de las partículas alargadas, estas producen concretos de poca trabajabilidad en estado fresco (Muhit et al., 2013). De acuerdo con la literatura. cambios en el tamaño máximo del agregado grueso afectan la resistencia del concreto (Mehta y Monteiro, 2006). Mezclas de concreto con un mayor tamaño de agregado grueso, para un mismo contenido de cemento y consistencia, requieren un menor contenido de agua en comparación con concretos con menor tamaño de agregado. De manera contraria, este incremento en el tamaño de agregado forma una débil zona de transición interfacial con mayor contenido de microgrietas. En concretos con alta y moderada resistencia a la compresión, el efecto de incrementar el tamaño máximo de agregado es mayor en comparación con concretos de bajas resistencia. Esto, debido a que una baja relación agua/cemento $(\mathrm{a} / \mathrm{c})$, reduce la porosidad de la zona de transición interfacial, que juega un papel importante en la resistencia mecánica. Por lo tanto, una reducción en el tamaño de agregado grueso, a una determinada relación a/c puede incrementar la resistencia a la compresión (Mehta y Monteiro, 2006).

La resistencia a la compresión es considerada la más importante propiedad en el diseño de estructuras de concreto, la cual es determinada usualmente a los 28 días de edad, bajo condiciones de temperatura y humedad controladas (Moradi et al., 2021; Irrigaray et al., 2016). En el diseño de concreto y control de calidad, la resistencia es la propiedad especificada. Muchas propiedades del concreto, como el módulo de elasticidad, impermeabilidad y la resistencia a agentes atmosféricos, se cree que dependen de esta propiedad (Mehta y Monteiro, 2006). Los factores que la afectan son: los agregados, el tipo de cemento, mezclado, transporte, colocación, compactación y curado (Gutiérrez de López, 2003).

Para construir estructuras de concreto reforzado durables que resistan la acción del tiempo o cualquier proceso de deterioro, es necesario conocer la calidad de los materiales (Hernández, 2017). En Nicaragua, la roca basáltica es la principal fuente de agregado grueso para la elaboración de concreto, y es comercializada en diferentes tamaños y calidades. El propósito de este estudio es evaluar el efecto del uso de agregado grueso de diferentes tamaños y calidades, sobre las propiedades mecánicas del concreto.

\section{PROCEDIMIENTO EXPERIMENTAL}

\subsection{Materiales}

Se emplearon dos tipos de cemento hidráulico de uso general (GU) que cumplen con lo indicado en la norma NTON 12 006-11 y la ASTM C1157 (ASTM, 2000), a los cuales se realizaron ensayos de tiempo de fraguado por medio de la aguja de Vicat, según la norma ASTM C 187 (ASTM, 2000) (Tabla 1). Se utilizó agregado grueso triturado de 3/4" y 1/2", en dos calidades (A y B), y arena de mina Motastepe. Sus propiedades físicas se muestran en la Tabla 2. La caracterización de los agregados se realizó según las normas ASTM C33, ASTM C70, ASTM C127, ASTM C128, ASTM C566 y ASTM C29 (ASTM, 2000).

Tabla 1. Caracterización del cemento

\begin{tabular}{lccc}
\hline Cemento & $\begin{array}{c}\text { Peso } \\
\text { específico } \\
\left(\mathrm{gr} / \mathrm{cm}^{3}\right)\end{array}$ & $\begin{array}{c}\text { Tiempo de } \\
\text { fraguado inicial } \\
(\mathrm{min})\end{array}$ & $\begin{array}{c}\text { Tiempo de } \\
\text { fraguado final } \\
(\mathrm{min})\end{array}$ \\
\hline $\begin{array}{l}\text { Cemento 1 } \\
(\mathrm{C} 1)\end{array}$ & 2.84 & 168 & 270 \\
$\begin{array}{l}\text { Cemento } 2 \\
(\mathrm{C} 2)\end{array}$ & 2.88 & 164 & 225 \\
\hline
\end{tabular}


Tabla 2. Propiedades físicas del agregado fino y grueso

\begin{tabular}{lccccc}
\hline Propiedades & Arena & Grava 1/2”A & Grava 1/2” B & Grava 3/4" A & Grava 3/4” B \\
\hline Tamaño máximo $(\mathrm{mm})$ & - & 12.50 & 12.50 & 19.00 & 19.00 \\
Densidad volumétrica $\left(\mathrm{kg} / \mathrm{m}^{3}\right)$ & 1491 & 1422 & 1485 & 1430 & 1354 \\
Gravedad específica & 2.82 & 2.88 & 2.84 & 2.85 & 2.78 \\
Absorción de agua $(\%)$ & 6.18 & 1.62 & 1.80 & 1.62 & 1.73 \\
Módulo de finura & 3.01 & - & - & - & - \\
\hline
\end{tabular}

\subsection{Preparación y curado de especímenes}

Se elaboraron 72 probetas de concreto con relación agua/cemento (a/c) de 0.50 (36 especímenes) y 0.45 (36 especímenes). El tamaño de los especímenes fue de $100 \mathrm{~mm}$ de diámetro x $200 \mathrm{~mm}$ de altura, y fueron curados en húmedo por 7 días. Se determinó la resistencia a la compresión y Velocidad de Pulso Ultrasónico (VPU), por triplicado. En la Tabla 3 se muestra la proporción de las mezclas de concreto y sus propiedades en estado fresco.

Tabla 3. Proporciones para la elaboración de $1 \mathrm{~m}^{3}$ de mezcla de concreto y propiedades en estado fresco

\begin{tabular}{lcccccccc}
\hline $\begin{array}{l}\text { Mezcla de } \\
\text { concreto }\end{array}$ & C1- 1/2A & C1- 1/2B & C2- 1/2A & C2- 1/2B & C1- 3/4A & C1- 3/4B & C2- 3/4A & C2- 3/4B \\
\hline a/c & 0.50 & 0.50 & 0.50 & 0.50 & 0.45 & 0.45 & 0.45 & 0.45 \\
Grava (kg) & 867 & 867 & 867 & 867 & 886 & 886 & 886 & 886 \\
Arena (kg) & 715 & 715 & 715 & 715 & 734 & 734 & 734 & 734 \\
$\begin{array}{l}\text { Cemento (kg) } \\
\text { Agua }(\mathrm{kg})\end{array}$ & 517 & 517 & 517 & 517 & 535 & 535 & 535 & 535 \\
$\begin{array}{l}\text { Revenimiento } \\
(\mathrm{cm})\end{array}$ & 263 & 263 & 263 & 263 & 242 & 242 & 242 & 242 \\
$\begin{array}{l}\text { Temperatura } \\
\left({ }^{\circ} \mathrm{C}\right)\end{array}$ & 34.0 & 34.0 & 34.5 & 34.0 & 36.0 & 34.0 & 34.0 & 34.5 \\
$\begin{array}{l}\text { Peso volumétrico } \\
\left(\mathrm{kg} / \mathrm{cm}^{3}\right)\end{array}$ & 2317 & 2300 & 2325 & 2279 & 2324 & 2295 & 2334 & 2303 \\
\hline
\end{tabular}

\subsection{Resistencia a la compresión}

La resistencia a la compresión fue determinada a los 28 y 180 días de edad, empleando una máquina de ensayo ELE Accu-Tek 250 con capacidad de 10,000 psi, de acuerdo con el procedimiento de ensaye descrito en la norma ASTM C39 (ASTM, 2000).

\subsection{Velocidad de pulso ultrasónico}

Se determinó la velocidad de pulso ultrasónico a los 28, 56 y 120 días edad. Se utilizó un equipo de pulso ultrasónico CONTROLS Mod. 58-E4800, con transductores de $54 \mathrm{KHz}$. Las mediciones de VPU fueron realizadas según el procedimiento indicado en la norma ASTM C597 (ASTM, 2000).

\section{RESULTADOS Y DISCUSIÓN}

En la Figura 1 se presentan los resultados de resistencia a la compresión axial de concretos con relación a/c $=0.50$ y grava de $1 / 2$ " (calidad A y B), a los 28 y 180 días de edad. A los 28 días, se observa que el valor de la mezcla C2-1/2A es mayor en comparación con las mezclas C1-1/2 y C2-1/2B. En los concretos C1-1/2, 
los valores de resistencia para la calidad A y B, fueron similares en ambas edades de prueba, indicando que la calidad del agregado no influye sobre los resultados. Los valores de resistencia de estos concretos incrementan con la edad.

A los 180 días, se observa que el valor de resistencia de las mezclas C1 y C2-1/2A fue similar. Se observa que en los concretos C2, la resistencia fue afectada por la calidad del agregado. En estas mezclas no se observan incrementos significativos de resistencia relacionados con la edad de prueba.

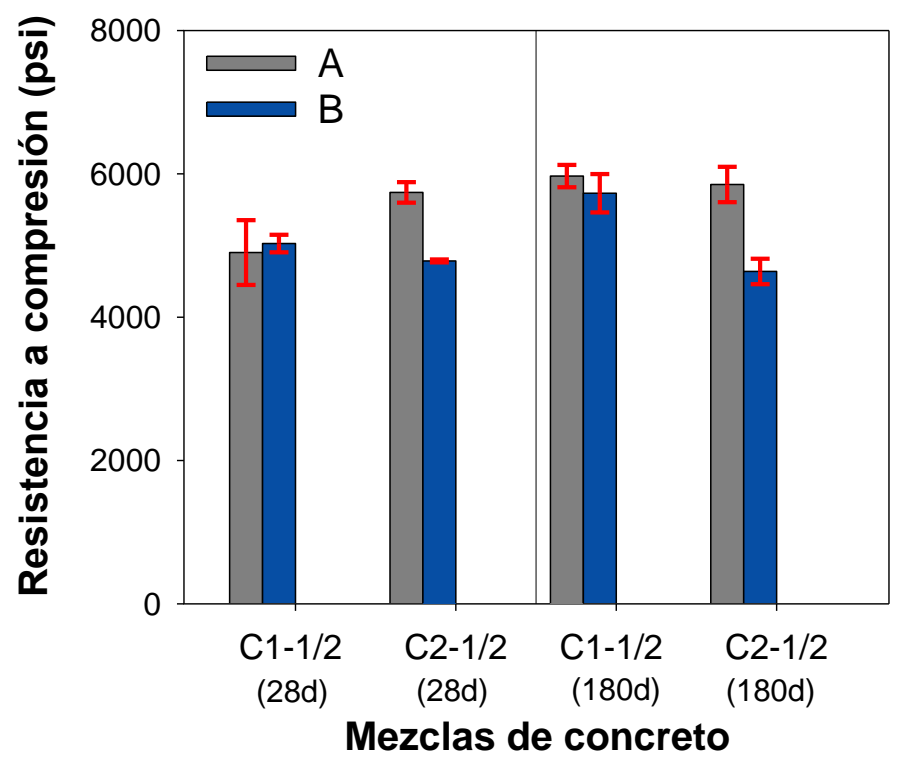

Figura 1. Resistencia a la compresión de especímenes de concreto con relación a/c de 0.50 y agregado de 1/2”, a los 28 y 180 días de edad. Las barras de error indican una desviación estándar.

En la Figura 2 se muestran los resultados de resistencia a la compresión axial de los concretos con relación a/c $=0.45$ y grava de 3/4" (calidad A y B), a los 28 y 180 días de edad. A los 28 días se observa que los valores de resistencia de las mezclas $\mathrm{C} 1-3 / 4$ y C2-3/4 fueron similares. La calidad del agregado no tuvo efectos significativos sobre los resultados.

A los 180 días, la mezcla C1-3/4B es la que presenta el mayor valor de resistencia a la compresión en comparación con el resto de concretos. En la Figura se observa que el valor de resistencia de los concretos C2-3/4 (A y B) fue similar, indicando que la calidad del agregado no tuvo afecto sobre los resultados. Se observa que únicamente en las mezclas $\mathrm{C} 1$ la resistencia incrementa con la edad.

En las Figuras 1 y 2, los valores de resistencia a la compresión de los concretos con agregado de 3/4" son mayores en comparación con los de $1 / 2$ ", como consecuencia de la menor relación a/c con que fueron elaborados. Según la literatura, en concretos, el uso de un mayor tamaño máximo de agregado grueso para un mismo contenido de cemento y consistencia, requiere una menor cantidad de agua de mezclado en comparación con concretos con agregado grueso de menor tamaño (Mehta y Monteiro, 2006). Por lo tanto, al reducir la relación a/c, se reduce la porosidad e incrementa la resistencia mecánica. 


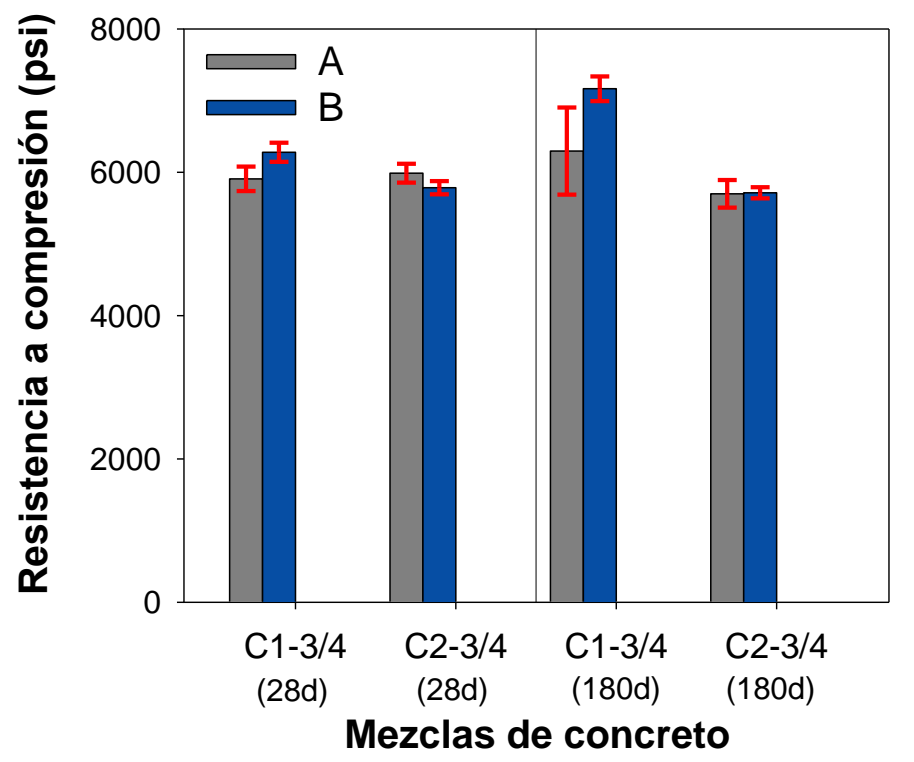

Figura 2. Resistencia a la compresión de especímenes de concreto con relación a/c de 0.45 y agregado de 3/4", a los 28 y 180 días de edad. Las barras de error indican una desviación estándar.

El uso de agregado de $3 / 4$ " no tuvo efectos negativos sobre los valores de resistencia mecánica de los concretos elaborados. El efecto de incrementar el tamaño del agregado grueso sobre la resistencia a la compresión es más pronunciado en concretos de alta resistencia en comparación con concretos de baja resistencia (Mehta y Monteiro, 2006). A los 180 días de edad, se observa que la resistencia de las mezclas con agregados de $1 / 2$ " y $3 / 4$ ", y calidad A, muestran valores de resistencia mecánica similares. Este resultado indica que la relación a/c de los concretos con agregado de 3/4" compensó el efecto negativo que puede generar el incremento de tamaño del agregado grueso. En concretos, el agregado grueso de mayor tamaño tiende a formar una zona de transición interfacial débil que contiene mayor micro-grietas, las cuales afectan la resistencia a la tensión y compresión (Mehta y Monteiro, 2006).

En la Figura 3 se presentan los resultados de la velocidad de pulso ultrasónico (VPU) de los concretos con relación a/c $=0.50$ y grava de 1/2" (calidad A y B), a los 28, 56 y 120 días de edad. Se observa que los valores de VPU de las mezclas C1 son mayores en comparación con los de las mezclas C2, para las tres edades de prueba. En las mezclas no se observan incrementos en los valores de VPU por la edad de prueba, ni por la calidad del agregado empleado. Los resultados de VPU de los concretos C1 muestran que son más densos en comparación con los concretos C2, por lo tanto, su resistencia mecánica es mayor (Irrigaray et al., 2016). El bajo valor de las barras de error indican que las mezclas fueron homogéneas. Estos resultados son consistentes con los observados en la Figura 1 a los 180 días de edad.

En la Figura 4 se presentan los resultados de la velocidad de pulso ultrasónico (VPU) de los concretos con relación a/c $=0.45$ y grava de 3/4" (calidad A y B), a los 28, 56 y 120 días de edad. Se observa que los valores de VPU de las mezclas C1 son mayores al de las mezclas C2, a los 56 y 120 días de edad. Los concretos con agregados calidad B presentan valores de VPU mayores en comparación con los de calidad A. Estos resultados indican que un mayor valor de VPU, representa una mayor densidad del material, por lo tanto, la resistencia mecánica de estos concretos también es mayor (Irrigaray et al., 2016). Estos resultados son consistentes con los observados en la Figura 2 a los 180 días de edad. 


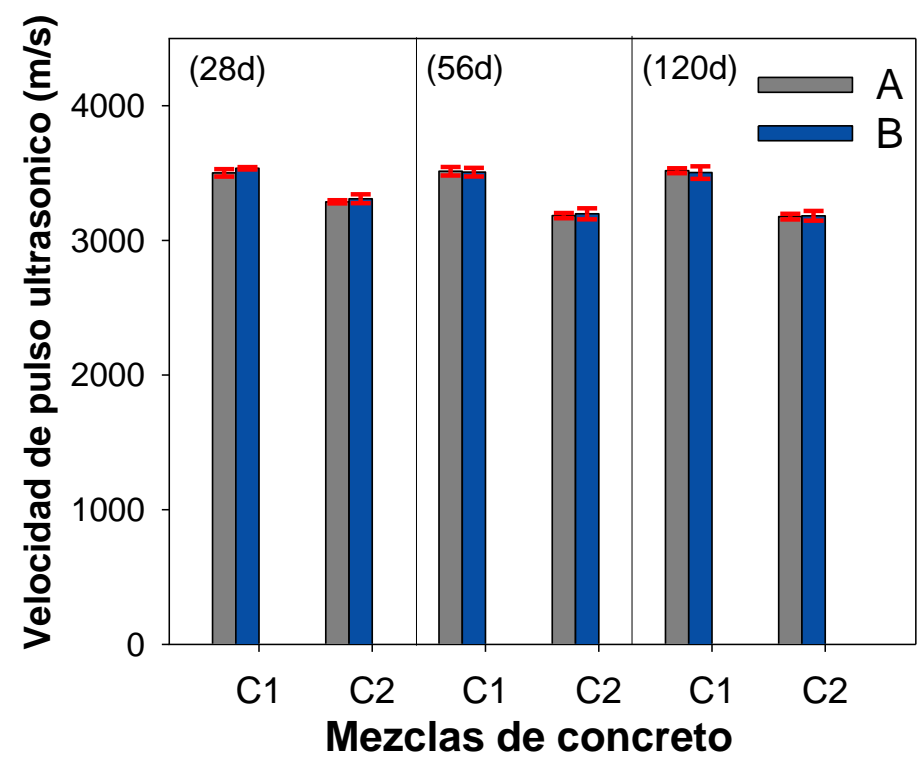

Figura 3. Velocidad de pulso ultrasónico de especímenes de concreto con relación a/c de 0.50 y agregado de 1/2”, a los 28, 56 y 120 días de edad. Las barras de error indican una desviación estándar.

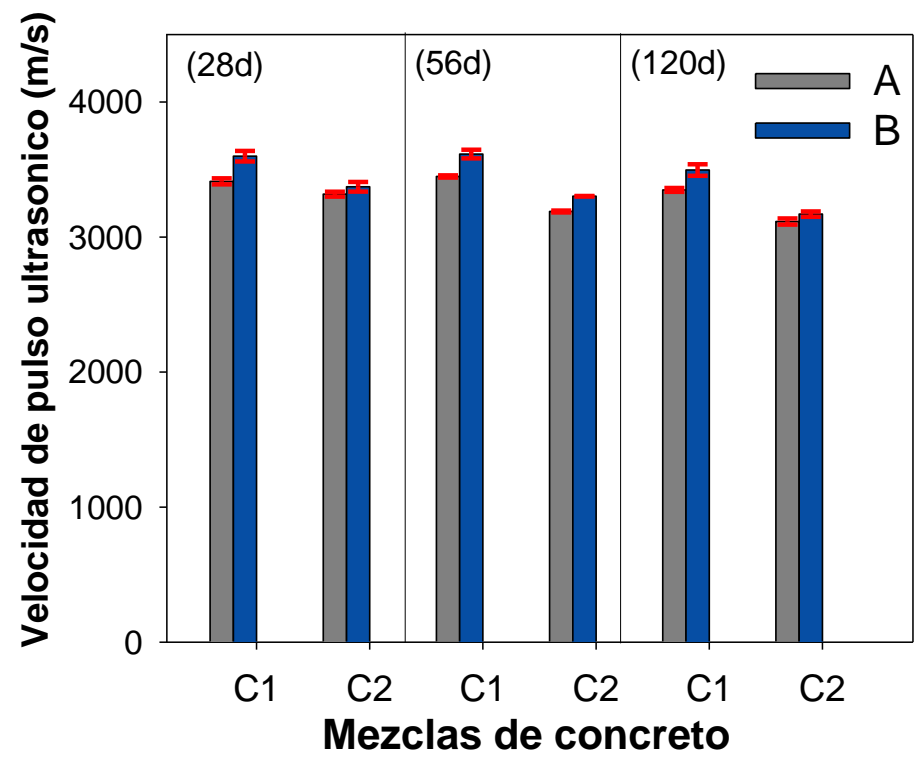

Figura 4. Velocidad de pulso ultrasónico de especímenes de concreto con relación a/c de 0.45 y agregado de 3/4", a los 28 y 56 y 120 de edad. Las barras de error indican una desviación estándar.

\section{CONCLUSIONES}

Con base en los resultados experimentales obtenidos se concluye que:

- El valor de revenimiento de los concretos con mayor tamaño de agregado se reduce al cambiar por efecto del tamaño de agregado la relación a/c, de 0.50 a 0.45 .

- En los concretos con agregado de 1/2", el tipo de cemento influyó sobre los resultados de resistencia a la compresión. A los 28 días, la resistencia de los concretos C2 calidad A, fue mayor que el resto 
de mezclas. A los 180 días, se observaron diferencias en los valores de resistencia por la calidad del agregado.

- En los concretos con agregado de 3/4", los valores de resistencia mecánica a los 28 días fueron similares. El tipo de cemento y la calidad del agregado no afectaron los resultados. A los 180 días, los concretos $\mathrm{C} 1$, presentaron valores de resistencia a la compresión mayores en comparación con los concretos $\mathrm{C} 2$.

- Los resultados de VPU fueron consistentes con los valores de resistencia a la compresión. Los concretos con mayor valor de VPU, presentaron mayores valores de resistencia a la compresión. En todas las mezclas el valor del error fue bajo, indicando que las mezclas son homogéneas.

\section{AGRADECIMIENTOS}

El autor agradece a la Vicerrectoría de Investigación y Desarrollo (VRID), de la Universidad Nacional de Ingeniería (UNI), de manera especial al Dr. Leonel Plazaola Prado, por la gestión realizada de los fondos de beca de apoyo para investigación e innovación 2019-2020 otorgados a este proyecto. También agradece al Dr. Oscar Gutiérrez, Decano de la Facultad de Tecnología de la Construcción (FTC) de la UNI, por su apoyo en las gestiones para el uso de los laboratorios de materiales de la FTC y TAISHIN. Así mismo, brinda especiales agradecimientos al MSc. Erasmo Aguilar Arriola por su incondicional apoyo, y a los estudiantes que colaboraron con el desarrollo de esta investigación.

\section{REFERENCIAS}

ASTM. (2000). Annual Bo-of ASTM Standards, Section Four, Construction, Volume 04.2 Construction, Concrete and Aggregates. ASTM.

Gutiérrez de López, L. (2003). El concreto y otros materiales para la construcción. In Paper Knowledge . Toward a Media History of Documents.

Hernández, E. F. (2017). Construcción durable - Una visión de futuro para Nicaragua. Arquitectura+, 2, 812.

Irrigaray, M. A. P., Pinto, R. C. de A., Padaratz, I. J. (2016). A new approach to estimate compressive strength of concrete by the UPV method. Revista IBRACON de Estruturas e Materiais, 9(3), 395-402. https://doi.org/10.1590/s1983-41952016000300004

Kosmatka, S. H., Kerkhoff, B., Panarese, W. C., Tanesi, J. (2004). Diseño y control de mezclas de Concreto. In Portland Cement Association (Primera, Vol. 1).

Mehta, K. P., Monteiro, P. J. . (2006). Concrete - Microstructure, properties and materials. In McGraw-Hill (Ed.), McGraw-Hill (Third, Issue 3). https://doi.org/10.1036/0071462899

Moradi, M. J., Khaleghi, M., Salimi, J., Farhangi, V., Ramezanianpour, A. M. (2021). Predicting the compressive strength of concrete containing metakaolin with different properties using ANN. Measurement: Journal of the International Measurement Confederation, 183(January), 109790. https://doi.org/10.1016/j.measurement.2021.109790

Muhit, I., Haque, S., Rabiul Alam, M. (2013). Influence of Crushed Coarse Aggregates on Properties of Concrete. American Journal of Civil Engineering and Architecture, 1(5), 103-106. https://doi.org/10.12691/ajcea-1-5-3 


\section{SEMBLANZA DE LOS AUTORES}

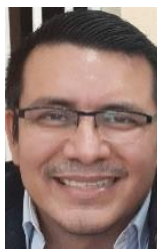

Eddisson Francisco Hernández: Es investigador acreditado de la Universidad Nacional de Ingeniería. Obtuvo el grado de Arquitecto en la Universidad Nacional de Ingeniería, Nicaragua, y los grados de maestría y doctorado en el Instituto Politécnico Nacional, México. Ha realizado estancias de investigación en la Universidad Nacional Autónoma de México y Universidad Marista de Querétaro, México. Sus intereses de investigación incluyen la ciencia y tecnología de materiales base cemento, así como la corrosión del acero de refuerzo en estructuras de concreto. 